\section{Fatores associados à incidência de maloclusão na dentição decídua em crianças de uma coorte hospitalar pública do nordeste brasileiro}

\section{Factors associated with the incidence of malocclusion in the deciduous dentition of children in a public hospital cohort from Northeast Brazil}

Suelly Pinto Teixeira de Morais 1

Eduardo Luiz Andrade Mota 2

Leila Denise A. F. Amorim 3

\begin{abstract}
Objectives: to estimate the incidence of malocclusion in deciduous dentition and the factors associated with it in early infancy.

Methods: a longitudinal study was carried out with children born in the city of Feira de Santana, Bahia, who were accompanied for 36 months. Incidence rates for malocclusion and incidence density ratios (IDR), with corresponding $95 \%$ confidence intervals, were obtained to assess the association between malocclusion and associated factors. Poisson regression models were adjusted to control for confounders.

Results: it was found that $34.4 \%$ of the children had mild malocclusion and $23.3 \%$ moderate or serious malocclusion, giving a total of $57.8 \%$ with some degree of malocclusion at three years of age. The overall incidence rate for malocclusion was 27.2 cases per 100 children per year. Children who used a pacifier or sucked their thumb had a higher risk of malocclusion than those who did not develop such sucking habits $(I D R=1.99 ; C I 95 \%=1.26-3.26)$. Children who were not exclusively or predominantly breastfed for a period of four months had a 1.5-fold greater risk of malocculsion $(C 195 \%=0.99-2.25)$ compared to children who were exclusively or predominantly breastfed during this period.

Conclusions: health workers are alerted to the need to encourage maternal breastfeeding and measures to prevent malocclusion in early infancy.
\end{abstract} Key words Malocclusion, Primary dentition, Breast feeding, Incidence, Risk factors

\author{
1 Universidade Federal do Recôncavo da Bahia. Avenida Carlos \\ Amaral, 1015. Cajueiro. Santo Antônio de Jesus, BA, Brasil. \\ E-mail: suelly.pinto@terra.com.br \\ 2 Instituto de Saúde Coletiva. Universidade Federal da Bahia, \\ Salvador, BA, Brasil. \\ 3 Instituto de Matemática. Universidade Federal da Bahia. Salvador, \\ BA, Brasil.
}

\section{Resumo}

Objetivos: estimar a incidência de maloclusão na dentição decídua e fatores associados na primeira infância.

Métodos: estudo longitudinal com crianças nascidas na cidade de Feira de Santana, Bahia, acompanhadas por 36 meses. Taxas de incidencia de maloclusão e razões de densidade de incidência (RDI), com correspondentes intervalos de $95 \%$ de confiança, foram obtidas para avaliar associação entre maloclusão e fatores associados. Modelos de regressão de Poisson foram ajustados para controle de confundimento.

Resultados: verificou-se que 34,4\% das crianças apresentaram maloclusão leve e 23,3\% maloclusão moderada ou grave, totalizando 57,8\% com algum grau de maloclusão aos três anos de idade. A taxa global de incidência de maloclusão foi de 27,2 casos por 100 crianças-ano. As crianças que sugaram chupeta ou dedo apresentaram risco de maloclusão maior quando comparadas com as crianças que não desenvolveram tais hábitos de sucção ( $R D I=1,99$; $I C 95 \%=1,26-3,26)$. Crianças que não foram amamentadas de forma exclusiva ou predominante por período igual a quatro meses tiveram um risco de maloclusão 1,5 vezes maior (IC95\%=0,99 - 2,25) quando comparadas com as crianças que mamaram de forma exclusiva ou predominante por este periodo.

Conclusões: alerta-se profissionais de saúde para a necessidade de estímulo ao aleitamento materno e medidas para prevenir as maloclusões na primeira infância.

Palavras-chave Maloclusão, Dentição decídua, Aleitamento materno, Incidência, Fatores de risco 


\section{Introdução}

As maloclusões são consideradas um problema de saúde pública sobretudo devido à sua expressiva prevalência e por acometerem crianças de pouca idade. ${ }^{1}$ O quadro epidemiológico de saúde bucal no Brasil apresenta níveis de precariedade que merecem atenção. As crianças brasileiras apresentam um dos mais altos índices de extrações dentárias prematuras, sem manutenção do espaço perdido. Lesões de cárie extensas não tratadas são fatores agravantes na determinação de maloclusões, que figuram na terceira posição na escala de prioridades e de problemas de saúde bucal no Brasil. ${ }^{2}$

Esses problemas podem ocasionar alterações nas funções de fonação e mastigação, desgastes dentários, alterações nos tecidos de suporte dos dentes e mesmo dores musculares, além de modificações estéticas que podem afetar a qualidade de vida, especialmente em se tratando de crianças e, uma vez presente em fase precoce, tendem a se agravar na dentição mista e permanente. 3

Diferentes fatores têm sido associados à ocorrência das maloclusões em crianças com dentição decídua, dentre os quais os comportamentais, hábitos de sucção não nutritivos, características antropométricas e nutricionais, padrões alimentares e suas alterações, uso de mamadeira e desmame precoce. $4-6$

Além da maloclusão estar associada a multifatores, algumas dificuldades em avaliar epidemiologicamente e comparar os resultados de inquéritos populacionais sobre os problemas oclusais incluem a multiplicidade de indicadores existentes, a grande variação nos critérios diagnósticos adotados, além de diferenças na composição etária, de gênero e etnia das populações.

A maioria dos estudos epidemiológicos identificados são de delineamento seccional e apresentam basicamente dois problemas que podem prejudicar ou enviesar seus resultados. O primeiro deles é a difícil separação entre causa e efeito já que a prevalência da doença e a exposição são mensurados simultaneamente nos estudos de corte transversal e, o segundo é o viés de memória, que pode acarretar no fornecimento de informações imprecisas, sobretudo em relação a questões comportamentais, dieta e hábitos de sucção não nutritivos ao nascimento e durante os primeiros anos de vida da criança. ${ }^{7}$ Diante do exposto, o objetivo deste estudo de delineamento longitudinal foi estimar a incidência de maloclusão na dentição decídua em uma coorte de crianças nascidas em um hospital público da Bahia e investigar os fatores comportamentais e socioeconômicos associados a este problema de saúde pública.

\section{Métodos}

Trata-se de um estudo longitudinal, prospectivo, realizado no Hospital Geral Clériston Andrade (HGCA), na cidade de Feira de Santana, Bahia, pertencente à rede pública estadual de serviços de saúde. O referido hospital possui maternidade referência para gravidez de alto risco com 27 leitos de alojamento conjunto e realiza, em média, 1500 partos/ano.

A coorte consistiu de crianças recém nascidas admitidas durante a sua permanência no alojamento conjunto, sendo estabelecida em julho de 2003, e as crianças foram seguidas até que completassem três anos de idade e a dentição decídua estivesse completa e em oclusão, sendo encerrada em junho de 2008. Foram incluídas as crianças cujas mães aceitaram participar da pesquisa, admitidas consecutivamente no alojamento conjunto, que assinaram termo de consentimento livre e esclarecido e que se enquadraram nos critérios de elegibilidade (residência em Feira de Santana, nascidas de parto natural ou cesáreo, gestação a termo e peso ao nascimento maior ou igual a 2500 gramas). Foram excluídas as crianças que tiveram intercorrências que impossibilitaram sua permanência no alojamento conjunto, as nascidas de parto a fórceps e aquelas que apresentavam alterações congênitas orofaciais diagnosticadas ao nascimento.

A coleta de dados teve início no alojamento conjunto e as demais avaliações foram feitas no ambulatório de puericultura. Foi criado um instrumento de pesquisa para registro dos dados demográficos, socioeconômicos, perinatais, comportamentais, além dos resultados do exame clínico.

O seguimento das crianças ao longo dos três anos foi feito da seguinte maneira: no primeiro ano foram examinadas ao nascimento e aos 2, 4, 6, 9 e 12 meses de vida; no segundo ano, aos 15, 18, $21 \mathrm{e}$ 24 meses e no terceiro ano, aos 30 e 36 meses, perfazendo um total de 12 exames ao longo do acompanhamento.

A variável dependente é a maloclusão (leve, moderada e severa) e as variáveis independentes incluem características comportamentais (hábitos de sucção de chupeta/dedo, tipo de aleitamento e dificuldade da mãe em amamentar a criança ao nascer) e sociodemográficas (escolaridade da mãe, renda da família, trabalho materno, paridade, tipo de parto e sexo da criança). Todas as variáveis sociodemográficas, além de dificuldade da mãe em amamentar a criança ao nascer, foram obtidas no início do acom- 
panhamento, ao nascimento. As demais variáveis comportamentais e nutricionais, tais como sucção de chupeta e sucção de dedo e o tipo de aleitamento, foram obtidas em cada uma das visitas durante os 36 meses de acompanhamento das crianças.

Aplicou-se neste estudo a categorização das maloclusões estabelecida pela Organização Mundial da Saúde (OMS), ${ }^{8}$ que classifica os tipos de oclusão como normal, maloclusão leve e maloclusão moderada ou grave. Estes critérios de classificação também foram adotados nos levantamentos nacionais de saúde bucal no Brasil.1,9

A condição oclusal aos 36 meses de vida das crianças com a dentição decídua completa foi classificada como: normal - quando não existia maloclusão nos primeiros 36 meses de vida; maloclusão leve - quando um ou mais dentes estavam em giroversão ou ligeiro apinhamento ou espaçamento prejudicando o alinhamento regular - e maloclusão moderada ou grave - quando havia um efeito inaceitável sobre a aparência facial, ou uma significativa redução da função mastigatória, ou problemas fonéticos observados pela presença de uma ou mais das seguintes condições nos quatro incisivos anteriores: a) trespasse horizontal maxilar estimado em $9 \mathrm{~mm}$ ou mais; b) trespasse horizontal mandibular, mordida cruzada anterior igual ou maior que o tamanho de um dente; c) mordida aberta; d) desvio de linha média estimado em $4 \mathrm{~mm}$ ou mais; e) apinhamento ou espaçamento estimado em $4 \mathrm{~mm}$ ou mais. Cabe ressaltar que as alterações oclusais não incluídas nos critérios mencionados, como, por exemplo, mordida cruzada posterior (uni ou bilateral), sobremordida ou trespasse vertical acima de 2 $\mathrm{mm}$, foram incluídas na categoria leve. ${ }^{8}$ Enfatizamos que os espaços interdentários localizados na seção anterior dos arcos decíduos não foram considerados como maloclusão, pois a presença destes espaços é fisiológica e altamente favorável na dentição decídua para o bom alinhamento dos dentes permanentes anteriores. 10

A oclusão foi avaliada em três planos: horizontal, vertical e transversal. Para a pesquisa da relação horizontal avaliou-se a sobressaliência, a relação entre os caninos e o plano terminal dos molares; para a investigação da relação vertical foram analisadas sobremordida e mordida aberta e, para a relação transversal foram observadas mordida cruzada e desvio de linha média.

Na relação horizontal, para avaliação da sobressaliência foi utilizada a classificação proposta por Foster e Hamilton 11 normal: a distância horizontal entre as bordas incisais superiores e inferiores não excede $2 \mathrm{~mm}$; nula: a sobressaliência equivale a zero; aumentada: sobressaliência maior que $2 \mathrm{~mm}$; ou negativa: os incisivos centrais inferiores localizam-se em uma posição vestibular aos incisivos centrais superiores. No exame dos relacionamentos entre os caninos decíduos também foram adotados os critérios propostos por aqueles autores ${ }^{11}$ : Classe 1, considerada relação normal; Classes 2 e 3 consideradas maloclusão. O relacionamento entre as superfícies distais dos segundos molares decíduos foi classificado de acordo com os critérios propostos por Baume 10: plano terminal reto, degrau mesial e degrau distal para a mandíbula, considerada como relação desfavorável.

Para a pesquisa da relação vertical, o sobrepasse foi classificado como normal quando era observado sobrepasse da borda incisal dos incisivos centrais decíduos inferiores até um terço da coroa; excessiva quando o sobrepasse da borda incisal dos incisivos centrais decíduos inferiores era maior que dois terços da coroa; e topo a topo quando não havia sobrepasse da borda incisal dos incisivos centrais decíduos inferiores pelos incisivos superiores. A mordida aberta anterior foi registrada caso existisse uma falta de sobreposição vertical entre quaisquer dos incisivos antagonistas, sem estimar a sua amplitude. 12

Na relação transversal, a mordida cruzada anterior e posterior foi registrada com os dentes em máxima intercuspidação habitual, quando um ou mais dentes superiores apresentassem uma inclinação axial anormal, ocluindo lingualmente em relação aos seus antagonistas. O desvio de linha média foi avaliado quando as faces mesiais dos incisivos superiores, em oclusão, não coincidiam verticalmente com as faces mesiais dos incisivos inferiores. 12

A maloclusão foi diagnosticada quando, na relação horizontal, o trespasse foi maior que $2 \mathrm{~mm}$, nulo ou negativo, os caninos em Classe 2 ou 3 e com relação terminal de molares em degrau distal para a mandíbula; na relação vertical, presença de mordida aberta e sobremordida excedendo um terço da coroa do incisivo inferior; na relação transversal, mordida cruzada anterior e/ou posterior e desvio de linha média.

O exame da cavidade bucal foi feito por meio de inspeção visual direta, uso de espelho bucal número 5 , espátulas de madeira descartáveis para afastar os tecidos moles e uso de régua plástica flexível milimetrada para a medida de sobressaliência. A régua plástica de 15 centímetros teve seu tamanho reduzido para 4 centímetros de comprimento por 0,5 centímetro de largura e as bordas foram arredondados com uso de lixa para que se adequasse 
às pequenas dimensões da cavidade bucal das crianças e para evitar traumatismos nos rodetes gengivais no momento da mensuração.

Os exames das crianças foram realizados no ambulatório de puericultura do HGCA, na companhia da genitora ou responsável, estando a criança deitada em posição supina, com a cabeça no mesmo nível do corpo, sob luz natural e luz fria. A examinadora se posicionou por trás da cabeça da criança, com as mãos enluvadas, sendo as luvas descartadas a cada exame; durante o exame clínico, foi feita a manipulação da mandíbula com os dedos anelares, até a posição de máximo fechamento afastando a língua da posição entre os rodetes gengivais e afastando também os tecidos moles. A régua milimetrada foi posicionada na porção média do rodete gengival inferior e medida a quantidade de trespasse horizontal do rodete superior. Após a erupção dentária, o ponto de referência para medir o tamanho do trespasse horizontal do arco superior com relação ao inferior foi a linha média entre os dentes incisivos centrais superiores e inferiores.

A sucção de chupeta ou dedo ( $\operatorname{sim} /$ não) foi classificada com relação à presença do hábito: aos 36 meses; em algum momento durante os 36 meses de acompanhamento; ou ainda ter sido observado por pelo menos quatro meses. As categorias de aleitamento utilizadas foram as preconizadas pela OMS13: aleitamento materno exclusivo, quando as crianças recebiam leite materno como única fonte de hidratação e alimentação; aleitamento materno predominante quando recebiam, além do leite materno, água, chás ou sucos, e em aleitamento materno complementado quando recebiam qualquer quantidade diária de leite materno, associado a outros alimentos. Considerou-se desmame precoce quando o aleitamento materno foi interrompido antes dos seis meses de vida. Para fins de análise considerou-se a situação do aleitamento aos quatro meses de vida, visto que a licença maternidade no Brasil na época do estudo era por este período. As categorias aleitamento exclusivo e predominante foram agrupadas.

Com o objetivo de reduzir as perdas de seguimento, a criança foi agendada para retornar, após cada exame, de acordo com sua faixa etária, tomando-se o cuidado de esclarecer aos pais ou responsável sobre a importância do retorno na data prevista. No caso da criança não retornar, inicialmente foi tentado o contato por telefone e, quando necessário, foi feita a visita domiciliar.

Foi realizado estudo piloto com um grupo de 20 crianças recém nascidas e suas genitoras com o objetivo de avaliar o instrumento de pesquisa, a técnica de coleta dos dados, o treinamento da entrevistadora e a calibração da examinadora, que realizou os exames clínicos subseqüentes, assistida por uma Auxiliar de Enfermagem. A calibração foi feita entre a examinadora e um cirurgião dentista mais experiente, doutor em ortodontia considerado como "padrão ouro". O cálculo da concordância intra e inter examinador foi realizado pelo coeficiente Kappa, que foi estimado com os dados de mensuração do trespasse antero posterior dos rodetes gengivais do grupo piloto em momentos distintos: ao nascimento e 30 dias após, com resultado de 0,85 .

O tamanho da amostra foi calculado com base nos seguintes parâmetros: frequência esperada de maloclusão na dentição decídua de $42 \%$, nível de confiança de $95 \%$, poder de $80 \%$ e razão de risco igual a 2 , obtendo-se um $n=154$ crianças.14-18 Considerando um percentual de perdas de seguimento de $30 \%$ ( $10 \%$ ao ano), obteve-se, ao final, uma amostra de 180 crianças.

Distribuições de frequências referentes às características oclusais são apresentadas. A taxa de incidência foi calculada dividindo-se o número de casos de maloclusão pelo número de crianças-ano de acompanhamento, que foram comparadas, segundo as variáveis de estudo, por meio da razão de densidade de incidência (RDI) e correspondente intervalo de $95 \%$ de confiança (IC).

A associação entre tipos de oclusão aos 36 meses e as variáveis sócio-demográficas e comportamentais foi avaliada através do teste qui-quadrado de independência $\left(\chi^{2}\right)$ ou teste Exato de Fisher, quando apropriado, considerando-se um nível de $5 \%$ de significância. Testes de tendência foram utilizados quando a variável de exposição era ordinal.

O modelo de regressão de Poisson foi ajustado para avaliar a associação entre maloclusão e os fatores associados, considerando-se o controle de potenciais variáveis de confusão. As razões de densidade de incidência (RDI) e os correspondentes intervalos de $95 \%$ de confiança estimados pelos modelos de regressão múltipla de Poisson são apresentados. Foram mantidas no modelo múltiplo apenas as variáveis que apresentaram valor de $\mathrm{p}$ igual ou superior a $20 \%$ na análise univariada. Utilizou-se AIC (Akaike Information Criterion) e BIC (Bayesian Information Criterion) como critérios estatísticos para escolha do modelo com melhor ajuste para estes dados. Para análise dos dados foi utilizado o programa estatístico STATA, versão $10^{\circledR}$.

Este estudo e o instrumento de coleta de dados foram submetidos inicialmente à avaliação do Comitê de Ética e Pesquisa da Universidade Estadual de Feira de Santana, Bahia (UEFS) sendo 
aprovada sob o Protocolo $n^{\circ}$. 030/2003 e posteriormente ao Comitê de Ética em Pesquisa do Instituto de Saúde Coletiva (ISC) - Universidade Federal da Bahia, aprovado sob o registro 042/2010.

\section{Resultados}

Não houve perda de seguimento. Todas as 180 crianças foram examinadas durante as 12 avaliações e acompanhadas desde o nascimento até os três anos de idade. Das 180 crianças estudadas, $57,0 \%$ eram do sexo masculino e $60,0 \%$ nasceram de parto natural. As mães destas crianças tinham idade média de 26 anos com desvio padrão de 6,7 anos; 46,7\% eram primíparas; $71,0 \%$ tinham renda familiar até um salário mínimo; 76,7\% não trabalhavam fora de casa; $61,7 \%$ cursaram o ensino fundamental e $38,3 \%$ o ensino médio.

A taxa global de incidência de maloclusão foi de 27,2 casos por 100 crianças-ano. Entre os 104 casos de maloclusão identificados, $62(59,6 \%)$ apresentaram maloclusão leve e $42(40,4 \%)$ maloclusão moderada ou grave, totalizando $57,8 \%$ de crianças com algum grau de maloclusão na dentição decídua aos três anos de idade.

O trespasse maxilo-mandibular das crianças ao nascimento no sentido antero-posterior variou de $0 \mathrm{a}$ $6 \mathrm{~mm}$, com média de $3 \mathrm{~mm}$ e desvio padrão de 1,4 $\mathrm{mm}$. Em relação aos rebordos gengivais no sentido vertical, observou-se em $89,4 \%$ das crianças uma relação de toque anterior e posterior, em $8,4 \%$ uma abertura anterior e toque posterior e em 2,2\% uma relação de topo. $\mathrm{Na}$ análise da condição oclusal observou-se, no plano horizontal, que 51,1\% tinham sobressaliência aumentada, com média de $3 \mathrm{~mm}$ e desvio padrão de $1,73 \mathrm{~mm}$; enquanto que a relação de caninos revelou $11,1 \%$ e $2,2 \%$ das crianças em maloclusão de Classe 2 e 3 , respectivamente. No plano vertical constatou-se que $18,9 \%$ das crianças apresentaram sobremordida negativa e $17,2 \%$ mordida aberta anterior e, no plano transversal, verificou-se que $5,5 \%$ das crianças apresentaram mordida cruzada e $6,1 \%$ desvio de linha média aos 36 meses de idade (Tabela 1).

A proporção de crianças com maloclusão moderada ou grave foi maior entre aquelas que usaram chupeta ou tinham o hábito de sucção de dedo aos 36 meses de idade $(71,1 \%)$, sucção de chupeta ou dedo alguma vez durante os 36 meses $(30,8 \%)$ e uso de chupeta ou dedo durante quatro ou mais meses $(35,8 \%)$, quando comparadas com aquelas que não tinham esses hábitos $(p<0,001)$. Houve também associação estatisticamente significante entre o tipo de aleitamento e condição oclusal
( $p=0,019)$, sendo a proporção de crianças com maloclusão moderada ou grave maior entre aquelas com desmame precoce $(45,5 \%)$ quando comparadas com crianças com outro tipo de aleitamento, 22,5\%; $16,0 \%$ e $21,6 \%$, respectivamente, para aquelas com aleitamento exclusivo, predominante e complementado aos quatro meses (Tabela 2).

Nenhuma das características sociodemográficas e do comportamento, que foram mensuradas ao nascimento, apresentaram efeito estatisticamente significativo na taxa de incidência de maloclusão. Apesar da redução de $13 \%$ na taxa de incidência de maloclusão dentre as crianças cuja renda das famílias é superior a 1 salário mínimo (SM) comparada com aquelas com renda familiar até $1 \mathrm{SM}$ ( $\mathrm{RDI}=0,87 ; 95 \% \mathrm{IC}=0,55-1,36$ ), a renda familiar não apresentou efeito significativo, ao nível de $5 \%$ (Tabela 3).

O hábito de sugar chupeta ou dedo ao final do acompanhamento foi um fator importante de risco. As crianças que apresentaram este hábito tiveram um risco (RDI) quase duas vezes $(\mathrm{RDI}=1,99$; $\mathrm{IC} 95 \%=$ 1,53-2,21) maior de desenvolver maloclusão quando comparadas com as crianças que não tiveram o hábito durante os primeiros 3 anos de vida. De maneira similar as crianças que mantiveram o hábito de sugar chupeta ou dedo por quatro ou mais meses tiveram risco 2,19 (IC95\%= 1,44-3,39) vezes maior de desenvolver maloclusão, respectivamente, quando comparadas com as crianças que não o fizeram (Tabela 4).

A taxa de incidência de maloclusão foi 49,0 e 24,9 por 100 crianças/ano, respectivamente, entre crianças que já tinham sido desmamadas aos quatro meses comparadas com aquelas que ainda mamavam, o que representa um risco 97\% (IC95\%= 1,11-3,30) maior de desenvolver maloclusão. Igualmente, as crianças que não foram amamentadas de forma exclusiva ou predominante aos quatro meses tiveram um risco de maloclusão $50 \%$ maior quando comparadas com as crianças que mamaram de forma exclusiva ou predominante até os quatro meses, $(\mathrm{RDI}=1,50$; IC95\%=0,99-2,25) (Tabela 4).

Considerando-se o ajuste simultâneo das variáveis referentes ao hábito de sucção não nutritivo (dedo ou chupeta) e ao tipo de aleitamento materno, apresentamos quatro modelos de regressão múltipla de Poisson definidos pela combinação dos fatores que afetaram significativamente a maloclusão (Tabela 5). De modo geral, mesmo após o ajuste pelo tipo de aleitamento materno, os hábitos de sucção não nutritivos, mensurados alguma vez durante os 36 meses (Modelos 1 e 2) ou por 4 ou mais meses (Modelos 3 e 4) mantiveram-se estatisti- 
camente significantes, com RDI variando de 1,82 a 2,09. O Modelo 4 é aquele que apresenta melhores critérios estatísticos em relação à bondade do ajuste (menores AIC e BIC).

\section{Tabela 1}

Distribuição percentual das características da condição oclusal das crianças nos planos horizontal, vertical e transversal, aos 36 meses de vida. Feira de Santana, BA, 2008.

\begin{tabular}{|c|c|c|}
\hline Condição oclusal & $N=180$ & $\%$ \\
\hline \multicolumn{3}{|l|}{ Plano horizontal } \\
\hline \multicolumn{3}{|l|}{ Sobressaliência } \\
\hline Normal & 76 & 42,2 \\
\hline Aumentada & 92 & 51,1 \\
\hline Negativa & 5 & 2,8 \\
\hline Topo a topo & 7 & 3,9 \\
\hline \multicolumn{3}{|c|}{ Relação dos caninos } \\
\hline Classe I & 156 & 86,7 \\
\hline Classe 2 & 20 & 11,1 \\
\hline Classe 3 & 4 & 2,2 \\
\hline \multicolumn{3}{|c|}{ Plano terminal dos molares } \\
\hline Reto & 21 & 11,7 \\
\hline Degrau mesial & 157 & 87,2 \\
\hline Degrau distal & 2 & 1,1 \\
\hline \multicolumn{3}{|l|}{ Plano vertical } \\
\hline \multicolumn{3}{|l|}{ Sobremordida } \\
\hline Normal & 139 & 77,2 \\
\hline Aumentada & 2 & 1,1 \\
\hline Negativa & 34 & 18,9 \\
\hline Topo a topo & 5 & 2,8 \\
\hline \multicolumn{3}{|l|}{ Mordida aberta } \\
\hline Ausente & 148 & 82,2 \\
\hline Anterior & 31 & 17,2 \\
\hline Posterior & 1 & 0,6 \\
\hline \multicolumn{3}{|l|}{ Plano transversal } \\
\hline \multicolumn{3}{|l|}{ Mordida cruzada } \\
\hline Ausente & 170 & 94,5 \\
\hline Anterior & 4 & 2,2 \\
\hline Posterior & 4 & 2,2 \\
\hline Total & 2 & 1,1 \\
\hline \multicolumn{3}{|c|}{ Desvio de linha média } \\
\hline Sim & 11 & 6,1 \\
\hline Não & 169 & 93,9 \\
\hline
\end{tabular}




\section{Tabela 2}

Associação entre tipo de oclusão e fatores relacionados com hábitos de sucção e alimentação aos 36 meses de vida. Feira de Santana, BA, 2008.

\begin{tabular}{|c|c|c|c|c|c|}
\hline \multirow{2}{*}{ Variáveis e categorias } & \multirow[b]{2}{*}{$N=180$} & \multicolumn{3}{|c|}{ Tipos de oclusão } & \multirow[b]{2}{*}{$p^{*}$} \\
\hline & & $\begin{array}{c}\text { Normal } \\
(\%)\end{array}$ & $\begin{array}{l}\text { Maloclusão } \\
\text { leve (\%) }\end{array}$ & $\begin{array}{c}\text { Maloclusão moderada / } \\
\text { grave (\%) }\end{array}$ & \\
\hline \multicolumn{6}{|c|}{$\begin{array}{l}\text { Sucção de chupeta ou dedo } \\
\text { aos } 36 \text { meses }\end{array}$} \\
\hline Sim & 38 & 7,9 & 21,1 & 71,1 & $<0,001$ \\
\hline Não & 142 & 51,4 & 38,0 & 10,6 & \\
\hline \multicolumn{6}{|c|}{$\begin{array}{l}\text { Sucção de chupeta ou dedo } \\
\text { alguma vez durante os } 36 \\
\text { meses }\end{array}$} \\
\hline Sim & 117 & 32,5 & 36,8 & 30,8 & $<0,001$ \\
\hline Não & 63 & 60,3 & 30,2 & 9,5 & \\
\hline \multicolumn{6}{|c|}{$\begin{array}{l}\text { Sucção de chupeta ou dedo } \\
\text { durante } 4 \text { ou mais meses }\end{array}$} \\
\hline Sim & 95 & 27,4 & 36,8 & 35,8 & $<0,001$ \\
\hline Não & 85 & 58,8 & 31,8 & 9,4 & \\
\hline \multicolumn{6}{|c|}{$\begin{array}{l}\text { Aleitamento exclusivo ou } \\
\text { predominante aos } 4 \text { meses }\end{array}$} \\
\hline Sim & 121 & 47,9 & 32,2 & 19,8 & 0,070 \\
\hline Não & 59 & 30,5 & 39,0 & 30,5 & \\
\hline \multicolumn{6}{|c|}{$\begin{array}{l}\text { Tipo de aleitamento aos } 4 \\
\text { meses }\end{array}$} \\
\hline Exclusivo & 71 & 47,9 & 29,6 & 22,5 & $0,019 * *$ \\
\hline Predominante & 50 & 48,0 & 36,0 & 16,0 & \\
\hline Complementado & 37 & 37,8 & 40,5 & 21,6 & \\
\hline Desmame & 22 & 18,2 & 36,4 & 45,5 & \\
\hline
\end{tabular}

*Teste qui-quadrado de independência; **Teste qui-quadrado de tendência. 
Tabela 3

Taxa de incidência de maloclusão* em crianças nos primeiros 36 meses de vida segundo fatores sociodemográficos e do comportamento ao nascimento. Feira de Santana, BA, 2008.

\begin{tabular}{|c|c|c|c|c|}
\hline Fatores & $N=180$ & $\begin{array}{l}\text { No de casos } \\
\text { de maloclusão } \\
(N=104)\end{array}$ & $\begin{array}{c}\text { Taxa de } \\
\text { incidência / } \\
100 \text { crianças- } \\
\text { ano }\end{array}$ & RDI $($ IC95\%)* * \\
\hline \multicolumn{5}{|l|}{ Sexo da criança } \\
\hline Masculino & 101 & 55 & 25,0 & $0,83(0,55-1,24)$ \\
\hline Feminino & 79 & 49 & 30,3 & \\
\hline \multicolumn{5}{|c|}{ Escolaridade materna } \\
\hline Superior & 69 & 41 & 28,6 & $1,04(0,65-1,69)$ \\
\hline Médio & 52 & 29 & 25,4 & $0,93(0,54-1,57)$ \\
\hline Fundamental & 59 & 34 & 27,4 & \\
\hline \multicolumn{5}{|c|}{$\begin{array}{l}\text { Renda da família (salário } \\
\text { mínimo) }\end{array}$} \\
\hline$>1$ & 53 & 29 & 24,7 & $0,87(0,55-1,36)$ \\
\hline$\leq 1$ & 127 & 75 & 28,3 & \\
\hline \multicolumn{5}{|c|}{ Trabalho materno } \\
\hline $\operatorname{Sim}$ & 38 & 19 & 22,4 & $0,77(0,44-1,27)$ \\
\hline Não & 138 & 84 & 29,2 & \\
\hline \multicolumn{5}{|l|}{ Paridade } \\
\hline$>2$ filhos & 52 & 29 & 25,1 & $0,89(0,56-1,38)$ \\
\hline$\leq 2$ filhos & 128 & 75 & 28,2 & \\
\hline \multicolumn{5}{|l|}{ Tipo de parto } \\
\hline Cirúrgico & 72 & 42 & 27,9 & $1,04(0,68-1.57)$ \\
\hline Natural & 108 & 62 & 26,8 & \\
\hline \multicolumn{5}{|c|}{ Dificuldade amamentar } \\
\hline Sim & 22 & 12 & 25,4 & $0,92(0,46-1,69)$ \\
\hline Não & 158 & 92 & 27,5 & \\
\hline
\end{tabular}

*Maloclusão leve, moderada ou grave; **RDI= Razão de Densidade de Incidência. 
Tabela 4

Taxa de incidência de maloclusão* em crianças nos primeiros 36 meses de vida segundo fatores comportamentais e amamentação. Feira de Santana, BA, 2008.

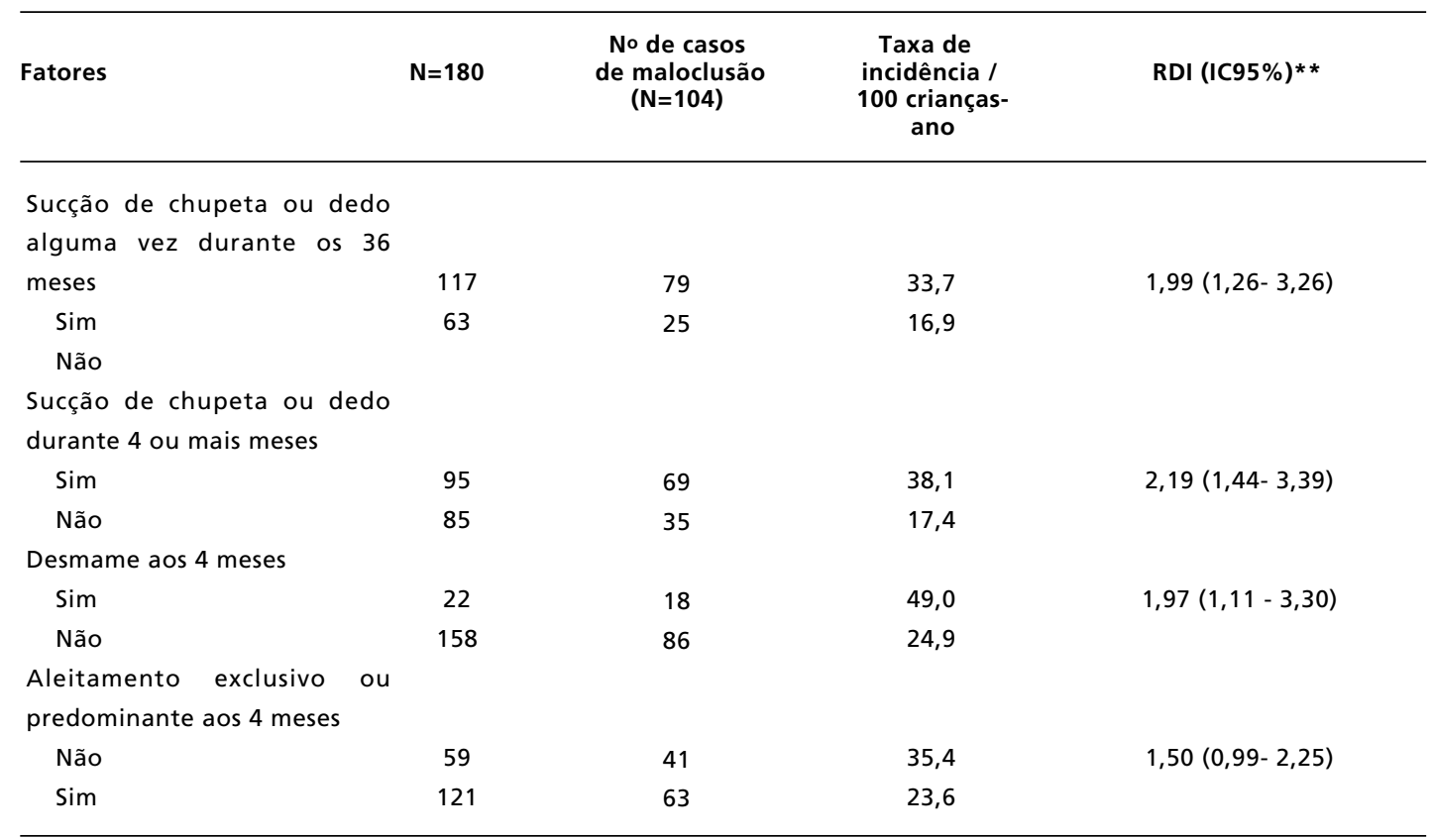

* Maloclusão leve, moderada ou grave.; **RDI= Razão de Densidade de Incidência.

Tabela 5

Associação entre maloclusão* em crianças e fatores comportamentais nos primeiros 36 meses de vida via modelos de regressão múltipla de Poisson. Feira de Santana, BA, 2008.

\begin{tabular}{lccc}
\hline Modelos/ Fatores & RDI (IC95\%)** & AIC & BIC \\
\hline $\begin{array}{l}\text { Modelo 1: } \\
\text { Sucção de chupeta/dedo alguma vez nos } 36\end{array}$ & $1,82(1,16-2,86)$ & & $-716,76$ \\
meses (ref***: não) & & \\
Desmame aos 4 meses (ref: não) & $1,59(1,02-2,48)$ &
\end{tabular}

Modelo 2:

Sucção de chupeta/dedo alguma vez nos 36

$1,90(1,22-2,95)$

meses (ref: não)

$1,36(0,96-1,93)$

Aleitamento exclusivo/predominante aos 4

meses (ref: sim)

Modelo 3:

Sucção de chupeta/dedo por 4 ou mais meses

$2,03(1,36-3,04)$

(ref: não)

$1,39(0,88-2,18)$

Modelo 4

$-720,76$

Sucção de chupeta/dedo por 4 ou mais meses

$2,09(1,42-3,07)$

(ref: não)

Aleitamento exclusivo/predominante aos 4

$1,29(0,91-1,83)$

meses (ref: sim)

AIC = Akaike Information Criterion; BIC = Bayesian Information Criterion; * Maloclusão leve, moderada ou grave; ** RDI= Razão de Densidade de Incidência; $* * *$ Categoria de referência (não tem hábito de sucção não nutritivo, tem aleitamento exclusivo ou predominante aos 4 meses; ou não desmamou aos 4 meses). 


\section{Discussão}

Os resultados deste estudo mostram que as maloclusões representam um problema de saúde bucal frequente e grave entre crianças com dentição decídua. Atitudes comportamentais, como o tempo de aleitamento materno, a presença e a frequência de hábitos bucais deletérios, foram os aspectos que mais contribuíram para a ocorrência das maloclusões.

Dentre as variáveis para as quais foi encontrada associação estatisticamente significante com a maloclusão, ressalta-se a sucção de chupeta ou dedo. Este estudo encontrou uma maior incidência de maloclusão entre aquelas crianças que usaram chupeta ou tinham o hábito de sucção digital. Estes achados também são corroborados por estudos de outros pesquisadores. 15,17,18,19-21 Segundo Moyers, 12 certos hábitos servem como estímulo ao crescimento normal dos maxilares, porém os hábitos anormais, como os de sucção de chupeta ou dedo, podem interferir no padrão regular de crescimento facial, podendo ser relacionados como fatores etiológicos do desenvolvimento de maloclusões. Graber22 destacou que a tríade: duração, intensidade e frequência do hábito de sucção não nutritivo pode influenciar o desenvolvimento craniofacial da criança, promovendo alterações neuromusculares, produzindo maloclusão e deglutição atípica.

O tipo de aleitamento também aparece como fator importante para a condição oclusal durante os primeiros 36 meses de vida. A literatura tem apontado a importância da sucção durante o aleitamento materno na promoção e no desenvolvimento adequado dos órgãos fonoarticulatórios quanto à mobilidade, força, postura e o desenvolvimento das funções de respiração, mastigação, deglutição e articulação dos sons da fala. ${ }^{23}$ Estudos sobre as práticas alimentares na primeira infância e sua relação com a instalação de hábitos de sucção e maloclusão na dentição decídua revelaram que a duração insuficiente do aleitamento materno, ou seja, o desmame precoce, está associado à presença de hábitos de sucção não nutritiva e estes, por sua vez, estão associados à ocorrência da maloclusão. Além da constatação de associação entre amamentação e hábitos de sucção, muitos pesquisadores também observaram menores percentuais de maloclusão nas crianças que foram amamentadas, ressaltando que quanto maior o tempo de amamentação, menor a presença de hábitos de sucção não nutritiva e maloclusão. $24-26$

Ressalta-se que, apesar de ter sido usada a metodologia da OMS (1987) para classificação da maloclusão na dentição decídua neste estudo, o que também havia utilizada pelo Projeto SB Brasil (2003), ${ }^{9}$ não se consideraram os espaços interdentários localizados na seção anterior dos arcos decíduos como maloclusão, pois a presença destes espaços é fisiológica e altamente favorável na dentição decídua para o bom alinhamento dos dentes permanentes anteriores. ${ }^{10}$ Esta é uma importante consideração afim de que não ocorram interpretações erronêas na classificação das maloclusões na dentição decídua.

Apesar de se tratar de uma amostra de conveniência, tornando o estudo não representativo da população-alvo, recomenda-se, com base nas evidências levantadas, que outros estudos longitudinais sejam realizados em outras populações para avaliação da magnitude do problema de maloclusão na infância.

Existe uma escassez na literatura de estudos sobre incidência de maloclusão na dentição decídua, o que dificultou uma análise comparativa com outros trabalhos de pesquisa em decorrência de desenhos de estudos díspares e critérios diferenciados de classificação das maloclusões na dentição decídua. Na literatura disponível no período analisado há uma variação na prevalência de maloclusão na dentiçao decídua de $30,0 \%$ a $80,2 \%$, indicando frequência elevada de maloclusão.14-18 No Brasil, os resultados do levantamento de saúde bucal realizado em 20039 mostraram que cerca de $36,5 \%$ das crianças de cinco anos de idade apresentaram maloclusão. No Nordeste, este percentual foi mais elevado, com $40,1 \%$ das crianças nesta faixa etária com esse problema. Em Salvador, na Bahia, o inquérito epidemiológico concluído em 2005, com a mesma metodologia do levantamento nacional, encontrou que $37,6 \%$ das crianças possuíam algum grau de maloclusão, 27 o que corrobora a alta proporção de crianças com maloclusão do nosso estudo, já que foi diagnosticada na dentição decídua de $57,8 \%$ das crianças com até três anos de idade.

A etiologia dos problemas oclusais relaciona-se a fatores primários, como os hereditários e alterações congênitas, e secundários, dentre os quais destacamse os hábitos bucais deletérios de sucção, como o uso de dedos e chupetas. Apesar de algumas investigações epidemiológicas terem concluído que os hábitos bucais deletérios sofrem influência das condições de vida familiares, mensuradas por fatores socioeconômicos, como renda, trabalho e escolaridade materna, 28,29 neste estudo não foram encontradas associações com tais fatores. Ainda são escassos e inconclusivos os estudos epidemiológicos que investigaram os determinantes dos hábitos 
bucais deletérios, o que pode limitar as ações preventivas dos serviços de saúde às recomendações normativas referentes aos prejuízos ocasionados por estes hábitos.

Sugere-se incluir medidas profiláticas e de promoção de saúde voltadas para crianças na primeira infância no planejamento e na organização dos programas e serviços públicos de saúde bucal, com uma perspectiva multiprofissional e interdisciplinar. É importante observar que os achados apontam para a necessidade de medidas promocionais e preventivas de âmbito familiar que

\section{Referências}

1. Brasil. Ministério da Saúde. Secretaria de Atenção à Saúde Departamento de Atenção Básica, Coordenação Naciona de Saúde Bucal. Projeto SB Brasil 2010. Brasília, DF; 2010

2. Tomita NE, Bijella VT, Franco LJ. The relationship between oral habits and malocclusion in preschool children. Rev Saúde Pública. 2000; 34 (3): 299-303.

3. Sipilä K, Zitting P, Siira P, Laukkanen P, Järvelin MR, Oikarinen KS, Raustia AM. Temporomandibular disorders, occlusion, and neck pain in subjects with facial pain: a case-control study. Cranio. 2002; 20 (3): 158-64

4. Massuia JM, Carvalho WO. Prevalence and associated factors of malocclusion in the primary dentition. RGO Rev Gaúcha Odontol. 2012; .60(3): 329-335.

5. Romero CC, Scavone-Junior H, Garib DG, Cotrim-Ferreira FA, Ferreira RI. Breastfeeding and non-nutritive sucking patterns related to the prevalence of anterior open bite in primary dentition. J Appl Oral Sci. 2011; 19 (2): 161-8.

6. Campos FL, Vazquez FL, Cortellazzi KL, Guerra LM , Ambrosano GMB, Meneghim MC, Pereira AC. The malocclusion and its association with socioeconomic variables, habits and care with five years old children. Rev Odontol UNESP. 2013; 42 (3): 160-6.

7. Almeida-Filho N, Barreto ML. Epidemiologia \& Saúde: Fundamentos, Métodos e Aplicações. Rio de Janeiro: Guanabara Koogan; 2012.

8. WHO (World Health Organization). Oral health surveys: basic methods. 3 ed. Geneva: ORH/EPID; 1987.

9. Brasil. Ministério da Saúde. Secretaria de Atenção à Saúde. Departamento de Atenção Básica, Coordenação Naciona de Saúde Bucal. Projeto SB Brasil 2003. Condições de saúde bucal da população brasileira 2002-2003. Resultados preliminares. Brasília, DF; 2004.

10. Baume LJ. Physiological Tooth Migration and its Significance for the Development of Occlusion. The biogenetic couce of the deciduos dentitions. J Dent Rev. 1950; 29 (2): 123-32.

11. Foster TD, Hamilton MC. Occlusion in the primary dentition: study of children at $21 / 2$ to 3 years of age. Br Dent $\mathrm{J}$ 1969; 126 (2): 76-81. reduzam os hábitos bucais, nocivos ao desenvolvimento satisfatório da oclusão decídua e, se necessário, intervenção em idade precoce para que estas maloclusões não se instalem ou se agravem na dentição mista e permanente. Medidas interdisciplinares, focadas no estímulo ao aleitamento materno e suas vantagens com relação ao crescimento e desenvolvimento da oclusão, também devem ser promovidas. Tais recomendações, porém, nem sempre são efetivas, visto que diversos fatores influenciam atitudes comportamentais.

12. Moyers RE. Ortodontia. 4 ed. Rio de Janeiro: Guanabara Koogan; 1991.

13. OMS (Organização Mundial da Saúde). Indicadores para evaluar las practicas de lactancia materna. Genebra: OMS/CED/SER; 1991.

14. Bezerra PKM, Cavalcanti AL. Características e distribuição das maloclusões em pré-escolares. Rev Ciênc Méd Biol. 2006; 5 (2): 117-23

15. Oliveira AB, Souza FP, Chiappetta ALML. Relação entre hábitos de sucção não nutritiva, tipo de aleitamento e má oclusões em crianças com dentição decídua. Rev CEFAC. 2006; 8 (3): 352-9.

16. Fernandes KP, Amaral MAT, Monico MA. Ocorrência de maloclusão e necessidade de tratamento ortodôntico na dentição decídua. RGO (Porto Alegre). 2007; 55 (3): 223-7.

17. Gimenez CMM, Moraes ABA, Bertoz AP, Bertoz F, Ambrosano GB. Prevalência de más oclusões na primeira infância e sua relação com as formas de aleitamento e hábitos infantis. Rev Dent Press Ortodon Ortop Facial. 2008; 13 (2): 70-83.

18. Fernandes KP, Amaral MT. Frequência de maloclusões em escolares na faixa etária de 3 a 6 Anos, Niterói, Brasil. Pesq Bras Odontoped Clin Integr. 2008; 8 (2): 147-51.

19. Bezerra PKM, Cavalcanti AL, Moura C. Maloclusões, tipo de aleitamento e hábitos bucais deletérios em pré escolaresum estudo de associação. Pesq Bras Odontoped Clin Integr. 2005; 5(3): 267-74

20. Furtado MAN, Vedovello MF. A influência do período de aleitamento materno na instalação dos hábitos de sucção não nutritivos e na ocorrência de maloclusão na dentição decídua. RGO (Porto Alegre). 2007; 55 (4): 335-41.

21. Mendes ACR, Valença AMG, Lima CCM. Associação entre aleitamento, hábitos de sucção não-nutritivos e maloclusões em crianças de 3 a 5 anos. Ciênc Odontol Bras. 2008; 11 (1): 67-75.

22. Graber TM. The "three M's": muscles, malformation, and maloccusion. Am J Orthod. 1962; 49 (6): 418-50.

23. Neiva FCB, Cattoni DM, Ramos JLA, Issler H. Desmame precoce: implicações para o desenvolvimento motor-oral. J Pediatr (Rio J.). 2003; 79 (1): 7-12. 
24. Souza DFRK, Valle MAS, Pacheco MCT. Relação clínica entre hábitos de sucção, má oclusão, aleitamento e grau de informação prévia das mães. Rev Dent Press Ortodon Ortop Facial. 2006; 11 (6): 81-90.

25. Almeida MEC, Melo NS, Maia SA, Costa AM, Souza KR A Influência do desmame precoce no desenvolvimento de hábitos bucais deletérios. ConScientiae Saúde, São Paulo. 2007; 6 (2): 227-34.

26. Kobayashi HM, Scavone H, Ferreira RI, Garib DG Relationship between breastfeeding duration and prevalence of posterior crossbite in the deciduous dentition. Am J Orthod Dentofacial Orthop. 2010; 137 (1): 54-8.

Recebido em 4 de julho de 2014

Versão final apresentada em 12 de agosto de 2014

Aprovado em 25 de setembro de 2014
27. Secretaria Municipal de Salvador - Bahia. Inquérito epidemiológico em saúde bucal, Salvador-BA, 2005. Resultados Principais. Salvador; 2006.

28. Tomita NE, Sheiham A, Bijella VT, Franco LJ. Relação entre determinantes socioeconômicos e hábitos bucais de risco para má-oclusão em pré-escolares. Pesq Odont Bras. 2000; 14 (2): 169-75.

29. Rossi TRA, Lopes LS, Cangussu MCT. Contexto familiar e alterações oclusais em pré-escolares de Salvador, Bahia, Brasil. Rev Bras Saúde Matern Infant. 2009; 9 (2): 139-47. 